\title{
Transtorno de estresse pós-traumático e a presença de recordações referentes à unidade de terapia intensiva
}

\author{
Posttraumatic stress disorder and the presence of \\ recollections from an intensive care unit stay \\ Jaquilene Barreto da Costa', Sonia Silva Marcon², Robson Marcelo Rossi ${ }^{3}$
}

\section{Palavras-chave}

Transtornos de estresse traumático, unidades de terapia intensiva, memória, amnésia.

\section{Keywords}

Stress disorders traumatic, intensive care unit, memory, amnesia.

\section{RESUMO}

Objetivo: Identificar a incidência de transtorno de estresse pós-traumático (TEPT) em pacientes submetidos à ventilação mecânica (VM) e analisar a sua relação com variáveis clínicas, demográficas e presença de recordações referentes à unidade de terapia intensiva (UTI). Método: Estudo prospectivo longitudinal realizado durante o período de um ano na UTI de um hospital-escola. A avaliação ocorreu três meses após a alta da UTI durante consulta ambulatorial. Os sujeitos foram avaliados utilizando-se um instrumento para avaliar memórias de UTI (IAM-TI) e o Impact of Event Scale-Revised (IES-R), para avaliar o TEPT. Resultados: Do total de respondentes, $68,8 \%$ eram do sexo masculino, a idade variou entre 18 e 79 anos, com média de 43,5 anos \pm 17,1. Quanto à avaliação dos pacientes, 14,5\% apresentaram sintomas de TEPT, quando utilizado um ponto de corte $>20$ pontos. Para uma melhor precisão diagnóstica, foi utilizado também o ponto de corte $\geq 33$ pontos e, nesse caso, sete pacientes $(5,1 \%)$ apresentaram o transtorno. Conclusão: Poucos pacientes preencheram todos os critérios para o diagnóstico de TEPT, indicando que a grande maioria deles apresentou apenas sintomatologia relacionada ao TEPT.

\section{ABSTRACT}

Objective: Identify the incidence of post-traumatic stress disorder (PTSD), in patients submitted to MV and analyze its relation with clinical and demographical variables, and with the presence of memories regarding intensive care unit (ICU). Method: It is a longitudinal prospective study, carried out in one year at an ICU of a university hospital. Evaluation occurred three months after ICU discharge during patients follow up at the ambulatory. Subjects were evaluated using the ICU memories (ICU-AMI) and the Impact of Event Scale-Revised (IES-R) instruments. Results: From the total of subjects, $68.8 \%$ were male from 18 to 79 years of age, 43.5 years old average \pm 17.1. From the evaluated patients, $14.5 \%$ presented symptoms of PTSD when a cut-off score $>20$ was used. For a diagnostic accuracy a $\geq 33$ cut-off score was also used, and in this case, only seven patients (5.1\%) showed signs of PTSD. Conclusion: A few patients met the criteria for the PTSD diagnosis indicating that the great majority of patients admitted at ICU show only symptomatology related to PTSD.

1 Hospital Universitário do Oeste do Paraná (HUOP), Setor de Psicologia.

2 Universidade Estadual de Maringá (UEM/PR), Departamento de Enfermagem.

3 UEM/PR, Departamento de Estatística.

Recebido em
$11 / 10 / 2011$

Aprovado em

$15 / 2 / 2012$
Endereço para correspondência: Jaquilene Barreto da Costa

Hospital Universitário do Oeste do Pará, Setor de Psicologia

Av. Tancredo Neves, 3224, Santo Onofre

85804260 - Cascavel, PR

E-mail: jaquihuop@yahoo.com.br 


\section{INTRODUÇÃO}

Consequências físicas e emocionais relacionadas à internação em unidade de terapia intensiva (UTI) têm despertado o interesse de muitos pesquisadores. Atualmente, é consenso entre os estudiosos da área ${ }^{1-4}$ que a internação em UTI pode gerar alterações emocionais por causa dos eventos estressantes e das diversas modalidades terapêuticas, a exemplo dos procedimentos invasivos, sedação, ruídos e privação do sono ${ }^{1,2}$.

Desordens mentais como ansiedade, depressão, alterações de memória e o transtorno de estresse pós-traumático (TEPT) têm sido amplamente estudadas, indicando que tais reações podem surgir após eventos traumáticos e estressantes, como a internação na UT $\left.\right|^{3-6}$.

Estima-se que a prevalência desse transtorno na população de pacientes internados em UTI geral varia entre 17\% e $30 \%{ }^{3-7}$. Já as taxas de incidência de TEPT apresentaram pouca variação em grupos específicos de UTI geral: 14\% a 18\% em pacientes cardíacos e $15 \%$ a $24 \%$ em pacientes com trau$m a^{6}$, diferença que pode ser possivelmente atribuída ao período da avaliação e aos diferentes instrumentos utilizados.

Alguns fatores de risco para o desenvolvimento de TEPT têm sido identificados, incluindo idade jovem ${ }^{7}$, gênero feminino ${ }^{2}$, longa permanência na UTI, altos níveis de sedação, história psiquiátrica anterior e presença de memórias ilusórias ${ }^{5,8,9}$.

O TEPT manifesta-se por meio de um conjunto de reações emocionais e comportamentais; seus sintomas causam sofrimento significativo em diversas áreas de funcionamento, como isolamento social e embotamento afetivo. Além disso, sua sintomatologia frequentemente está associada às comorbidades psiquiátricas, como os transtornos de ansiedade, depressão, transtorno de pânico e dissociação,10.

Para diagnosticar o TEPT, o paciente deve ter sido exposto a um evento estressante de curta ou longa duração. Em consequência desse evento, desenvolvem-se três dimensões de sintomas: 1) Revivência - caracteriza-se por recordações aflitivas recorrentes e intrusivas do evento, incluindo pensamentos, imagens ou percepções, sonhos recorrentes, agir ou sentir como se o evento traumático estivesse ocorrendo novamente com episódios de flashbacks; 2) Esquiva/ entorpecimento emocional - pensamentos de evitação associados ao trauma; incapacidade de lembrar algum aspecto importante do trauma e sentimento de distanciamento das outras pessoas; 3) Hiperestimulação/hipervigilância - caracterizado pela dificuldade em conciliar ou manter o sono, irritabilidade ou surtos de raiva, dificuldade em concentrar-se, hipervigilância e resposta de sobressalto exagerada ${ }^{10,11}$.

Os sintomas do TEPT habitualmente aparecem depois de um mês e até três meses após o evento traumático, configurando-se um quadro agudo, e se a duração é superior a esse período, é diagnosticado como crônico. Quando o início dos sintomas ocorre em um período superior a seis meses, especifica-se TEPT com início tardio ${ }^{12}$.
A observação de que as vivências do paciente e os tipos de memórias referentes à sua permanência na UTI podem desencadear alterações emocionais a longo e curto prazo são temas devidamente documentados ${ }^{13,14}$. Estudos sobre $\mathrm{o}$ TEPT em pacientes avaliados após a alta da UTI classificaram as memórias do período de internação em memórias de fatos reais, memórias de sentimentos e memórias ilusórias 5,6,15. As memórias ilusórias são memórias de formações delirantes e oníricas formadas após a retirada da sedação, no momento de recuperação da consciência ${ }^{15}$.

Recordações de sonhos, pesadelos e alucinações, além das experiências vividas na UTI, são frequentemente relatadas pelo paciente após a alta. Tais recordações podem ser de natureza desagradável e persistente ao longo do tempo, estando associadas ao risco de desenvolver reações atípicas crônicas, como o TEPT5,6,9,15,16.

Nos últimos anos, os cuidados intensivos têm sido vistos como um processo que envolve o bem-estar psíquico do indivíduo após a alta. Assim, considerando o impacto da internação na UTI para a saúde mental do paciente, a avaliação e a identificação precoce das possíveis alterações têm se configurado como relevantes e de considerável importância clínica.

Diante disso, definiu-se como objetivo do estudo identificar a incidência do TEPT em pacientes submetidos à ventilação mecânica (VM) e analisar a sua relação com variáveis clínicas, demográficas e presença de recordações referentes à UTI.

\section{MÉTODO}

Trata-se de um estudo prospectivo longitudinal, com abordagem quantitativa, realizado durante o período de um ano na UTI de um hospital-escola localizado na região oeste do Paraná. A UTI em estudo é referência de alta complexidade para 26 municípios da região e conta com nove leitos destinados a pacientes adultos acometidos de patologias clínicas diversas.

Participaram do estudo todos os pacientes internados durante o período de setembro de 2008 a agosto de 2009 que preencheram os seguintes critérios de inclusão: 1) maiores de 18 anos; 2) com permanência maior que 24 horas na UTI; 3) que ficaram em VM. Optou-se por estudar apenas os pacientes sob VM, por eles constituírem a maioria dos pacientes internados na UTI em estudo e também para tornar a amostra mais homogênea em virtude das características e especificidades dos procedimentos realizados com esses pacientes, o que é bastante diverso do que ocorre com aqueles que não são submetidos a VM.

Foram excluídos pacientes com diagnóstico prévio de doença mental, doença terminal, os internados por tentativa de suicídio, os impossibilitados de falar, os que apresentaram 
danos neurológicos após a alta da unidade e aqueles que não retornaram ao serviço para atendimento/acompanhamento ambulatorial.

A primeira etapa de coleta de dados ocorreu durante o período da internação do paciente na UTI. Para tanto, realizou-se diariamente o rastreamento das admissões e alta de pacientes por meio da verificação do livro de registro da unidade. Em seguida, a partir de consulta ao prontuário, foram identificados aqueles pacientes que atendiam aos critérios de inclusão e para estes foram coletados dados sociodemográficos e clínicos, incluindo causa da admissão, complicações na UTI, presença de agitação e confusão mental, tempo de ventilação mecânica, sedação e tempo de permanência na UTI.

A avaliação ocorreu três meses após a alta da UTI por ocasião do retorno do paciente no Ambulatório Interdisciplinar de Seguimento em Terapia Intensiva da instituição pesquisada. Nessa ocasião, foram aplicados dois instrumentos: 1) instrumento para avaliar memórias da UTI (IAM-UTI) ${ }^{17}$; e 2) Impact of Event Scale-Revised (IES-R) ${ }^{18}$, para avaliar estresse pós-traumático. Para confirmação do diagnóstico, foram utilizados dados de prontuário referentes à entrevista psicológica realizada com o paciente na avaliação ambulatorial. A entrevista psicológica utilizou os critérios diagnósticos empregados no Manual de Diagnóstico e Estatística das Perturbações Mentais (DSM-IV-R).

Para os pacientes que não compareceram à consulta ambulatorial, ocasião em que seria realizada a segunda etapa da coleta de dados, foi realizada busca ativa por meio de contato telefônico e agendada nova data para a realização dela. Em caso de não comparecimento, o paciente foi excluído do estudo. Os motivos apontados para o não comparecimento à consulta agendada foram: a) dificuldade de transporte, uma vez que um número razoável de pacientes residia em outros municípios; b) dificuldade de algum familiar acompanhar o paciente especialmente nos casos de idosos; e c) condições clínicas do paciente - acamado e/ou com dificuldade de locomoção, entre outras.

\section{Instrumentos}

1. Planilha para coleta de dados do prontuário. Nesta foram registrados dados sociodemográficos (nome, idade, sexo, escolaridade, estado civil) e clínicos (tempo de VM, de permanência na UTI e enfermaria, dados de sedação, causa de admissão e complicações na UTI). Quanto à prática de sedação, foram coletados do prontuário, durante a internação do paciente na UTI, os seguintes dados: a) sedação e analgesia: tipo de droga, tempo de sedação em dias, dose diária administrada, quantidade total administrada; b) nível de sedação: dados referentes às escalas de RASS (Escala de Richmond de Agitação-Sedação); c) registro da presença de agitação, confusão mental e uso de restrição física. Para classificação dos níveis de sedação, utilizou-se como parâmetro a seguinte classificação: pontuação de -2 e -3 (sedação leve a moderada) e -4 e -5 (sedação profunda). A escala de RASS é baseada em critérios clínicos, com numeração variando entre +4 e -5 pontos para graduar ansiedade, agitação ou ambas até estado de coma irresponsivo.

2. Instrumento de avaliação de memórias de UTI'17. Elaborado especificamente para o estudo com base no conteúdo de alguns itens da versão inglesa do instrumento Intensive Care Unit Memory (ICUM) ${ }^{19}$. O instrumento ora proposto foi validado com a ajuda de 11 experts da área de terapia intensiva e os resultados mostraram que a maioria dos itens foi avaliada de forma positiva, alcançando alta concordância entre os peritos. O referido instrumento é do tipo checklist constituído por duas questões abertas e nove itens agrupados em quatro grandes domínios: memórias relacionadas ao tratamento (tubo no nariz e boca, extubação, aspiração etc.); memórias do ambiente (visita da família, barulho, alta para enfermaria etc.); memórias relacionadas às experiências emocionais (dor, sede, fome, agitação, confusão, medo etc.) e àquelas relacionadas às memórias ilusórias (sonhos, pesadelos e alucinações). Para identificação dos tipos de memórias, classificou-se como apresentando amnésia total - aqueles pacientes que não tiveram nenhuma recordação do período de internação na UTI; memórias de fatos reais - aqueles com recordações relacionadas a fatos reais referentes ao tratamento, ao ambiente e eventos emocionais; e, por fim, memórias ilusórias - relacionadas a eventos irreais, como pesadelos, sonhos e alucinações.

3. Impact of Event Scale-Revised (IES-R) ${ }^{18}$. A escala contém 22 itens agrupados em três subescalas. A primeira subescala está relacionada com sintomas de intrusão (sete itens), caracterizada pela presença de pensamentos e imagens indesejadas. A segunda é caracterizada pela presença de pensamentos de evitação (oito itens). E por último a subescala de hiperestimulação (sete itens), que inclui distúrbios do sono, sintomas de flashback e labilidade emocional. As respostas são pontuadas numa escala tipo likert de cinco pontos e o seu escore varia de 0 a 66 pontos. Um escore $>20$ indica a presença de sintomas de TEPT e uma pontuação $\geq 33$ indica provável transtorno clínico ${ }^{20}$. A IES-R tem demonstrado boa consistência interna, validade preditiva e discriminativa ${ }^{21}$.

\section{Análise estatística}

Os dados foram armazenados no programa Microsoft Excel ${ }^{\oplus}$ e para análise e interpretação dos resultados foram utiliza- 
dos os programas Statistica 8.0 e Statistical Analisys System (SAS-9.1). Para comparar os grupos com e sem sintomas de TEPT, as seguintes variáveis foram categorizadas como independentes: sexo, idade, causa de admissão, tempo de UTI, tempo de ventilação mecânica, recordações de UTI e tipos de memórias. O Teste Exato de Fisher foi empregado para investigar a associação de cada fator com o desfecho. Estatísticas descritivas foram realizadas para todas as variáveis, visando à caracterização geral dos participantes e à descrição da presença de recordações referentes à UTI. Os resultados são mostrados em forma de tabelas e apresentados em frequência absoluta e relativa. Dados contínuos são apresentados como média e desvio-padrão quando indicados e dados categóricos, em número absoluto e porcentagem. Em todas as análises foi estabelecido o nível de significância de 5\%.

$\mathrm{O}$ estudo foi desenvolvido em conformidade com o preconizado pela Resolução no 196/96 do Conselho Nacional de Saúde, e o projeto foi apreciado pelo Comitê Permanente de Ética em Pesquisas envolvendo Seres Humanos da Universidade Estadual de Maringá (Parecer no 461/2008). A avaliação de todos os pacientes incluídos no estudo ocorreu após a assinatura do termo de consentimento livre e esclarecido.

\section{RESULTADOS}

Ao todo, 330 pacientes foram admitidos na UTI durante o período do estudo. Desses, 279 atenderam aos critérios de inclusão e 138 foram avaliados no seguimento três meses após a alta da UTI. Os demais deixaram de ser avaliados por motivos diversos como: óbito durante a internação (78) e após a alta (8); sequelas neurológicas, confusão mental, traqueostomia e quadro clínico grave (32); transferência (3); alta hospitalar para casa (4); não comparecimento para avaliação no ambulatório (16).

Dos 138 participantes, 68,8\% eram do sexo masculino, a idade variou entre 18 e 79 anos, com média de 43,5 anos $( \pm 17,1)$. A maioria dos pacientes $(44,2 \%)$ foi admitida por causa de trauma, seguidos daqueles com disfunções clínicas diversas $(42,1 \%)$ e por condições neurológicas $(13,7 \%)$. O tempo médio de permanência na UTI foi de 10,0 dias $( \pm 8,5)$, variando entre 2 e 37 dias, e $47,8 \%$ dos pacientes ficaram internados na UTI por mais de sete dias e mais da metade $(55,8 \%)$ permaneceu em ventilação mecânica por mais de dois dias, com um tempo médio de 7,2. Características demográficas e clínicas de acordo com a presença ou não de sintomas de TEPT são mostradas na tabela 1.

Quanto à presença do TEPT, 14,5\% dos pacientes apresentaram sintomas de TEPT quando foi utilizado um ponto de corte $>20$. Para melhor precisão diagnóstica, foi utilizado também o ponto de corte $\geq 33$ pontos e, nesse caso, somente sete pacientes $(5,1 \%)$ apresentaram o transtorno clínico, confirmado com dados da entrevista psicológica realizada no ambulatório. Dos sete pacientes que apresentaram TEPT, cinco eram mulheres, com idade média de 38,7 anos, com tempo de UTI e VM maior que dois dias e tempo médio de internação na enfermaria de 21,5 dias. Todos os pacientes com TEPT apresentaram alteração psicológica - cinco apresentaram sintomas de ansiedade e dois, ansiedade e depressão.

Não foram observadas associações entre presença ou não de TEPT e variáveis demográficas e clínicas, com exceção da faixa etária abaixo dos 50 anos de idade $(p=0,0496)$. Embora os resultados das análises estatísticas não tenham apontado associação entre os grupos, constatou-se no grupo de pacientes que apresentou sintomas do TEPT um tempo de internação maior do que sete dias e de VM maior do

Tabela 1. Características demográficas e clínicas de acordo com a presença ou não de sintomas do TEPT $(n=138)$ (Cascavel, $\mathrm{PR} / 2008-2009)$

\begin{tabular}{|c|c|c|c|}
\hline \multirow[t]{2}{*}{ Variáveis } & \multicolumn{3}{|c|}{ Sintomas de TEPT } \\
\hline & $\operatorname{Sim}(n=20)$ & Não (n= 118) & \\
\hline & $\mathrm{n}(\%)$ & $\mathrm{n}(\%)$ & $\mathrm{p}$ \\
\hline \multicolumn{4}{|l|}{ Sexo } \\
\hline Masculino & $11(55,0)$ & $84(71,2)$ & 0,1483 \\
\hline Feminino & $9(45,0)$ & $34(28,8)$ & \\
\hline \multicolumn{4}{|l|}{ Faixa etária } \\
\hline Até 50 anos & $19(95,0)$ & $89(75,4)$ & 0,0496 \\
\hline Acima de 50 anos & $1(5,0)$ & $29(24,6)$ & \\
\hline \multicolumn{4}{|l|}{ Causa da admissão } \\
\hline Trauma & $10(50,0)$ & $51(43,2)$ & 0,5369 \\
\hline Clínica & $9(45,0)$ & $49(41,5)$ & \\
\hline Neurológica & $1(5,0)$ & $18(15,3)$ & \\
\hline \multicolumn{4}{|l|}{ Tempo de UTI } \\
\hline Até $48 \mathrm{~h}$ & $2(10,0)$ & $17(14,4)$ & 1,0000 \\
\hline 3-7 dias & $8(40,0)$ & $45(38,1)$ & \\
\hline$>7$ dias & $10(50,0)$ & $56(47,5)$ & \\
\hline \multicolumn{4}{|l|}{ Tempo de ventilação mecânica } \\
\hline$\leq 2$ dias & $7(35,0)$ & $53(44,9)$ & 0,5043 \\
\hline$>2$ dias & $13(65,0)$ & $65(55,1)$ & \\
\hline \multicolumn{4}{|l|}{ Sedação } \\
\hline Sim & $13(65,0)$ & $79(66,9)$ & 0,8231 \\
\hline Não & $7(35,0)$ & $39(33,1)$ & \\
\hline \multicolumn{4}{|l|}{ Tempo de sedação } \\
\hline Sem sedação & $7(35,0)$ & $39(33,1)$ & 0,6756 \\
\hline$<2$ dias & - & $11(9,3)$ & \\
\hline $2-7$ dias & $6(30,0)$ & $40(33,9)$ & \\
\hline$>7$ dias & $7(35,0)$ & $28(23,7)$ & \\
\hline \multicolumn{4}{|l|}{ Nível de sedação } \\
\hline Sem sedação & $7(35,0)$ & $38(32,2)$ & 0,3365 \\
\hline Leve-moderada & - & $13(11,0)$ & \\
\hline Profunda & $13(65,0)$ & $67(56,8)$ & \\
\hline \multicolumn{4}{|l|}{ Memórias da UTI } \\
\hline Sim & $16(80,0)$ & $89(75,4)$ & 0,7185 \\
\hline Não & $4(20,0)$ & $29(24,6)$ & \\
\hline \multicolumn{4}{|l|}{ Tipos de memórias } \\
\hline Sem memória & $4(20,0)$ & $28(23,7)$ & 0,4410 \\
\hline Memórias de fatos reais & $6(30,0)$ & $51(43,2)$ & \\
\hline Memórias ilusórias & - & $4(3,4)$ & \\
\hline Memórias reais e ilusórias & $10(50,0)$ & $35(29,7)$ & \\
\hline
\end{tabular}


que dois dias (50,0\% e 65,0\%, respectivamente), mais da metade dos pacientes foi sedada $(65,0 \%)$ e submetida à sedação profunda (65,0\%), e a grande maioria relatou memórias da UTI (80,0\%).

Ao se investigar de forma mais específica os sintomas do TEPT, observa-se na tabela 2, a partir dos escores alcançados por cada um dos domínios que compõem o instrumento IES-R, que o maior valor médio entre os domínios esteve relacionado a pensamentos de evitação $(5,2)$ e com maior variabilidade de resultados ( $D P=4,90$ ), observando-se, também, que nesse domínio os pacientes alcançaram a maior pontuação quando comparado aos outros. Na sequência ficou o agrupamento de sintomas de intrusão (3,9 pontos), seguido da subescala de hiperestimulação (3,6 pontos).

Pelas medidas descritivas referentes aos domínios do instrumento usado para avaliar memórias de UTI, constatou-se que as médias de recordações relacionadas a fatos reais foram relativamente próximas $(5,2 ; 5,1$ e 5,1), conforme mostrado na tabela 2.

Tabela 2. Medidas descritivas dos valores obtidos para cada domínio do instrumento IES-R e IAM-UTI (Cascavel, PR/2008-2009)

\begin{tabular}{lccc}
\hline \multicolumn{1}{c}{ Domínios } & $\mathbf{N}(\%)$ & Média/DP & Variação \\
\hline IES-R $\left(\mathbf{n}=\mathbf{2 6 0}^{*}\right)$ & & & \\
Evitação & $82(31,5)$ & $5,2 \pm 4,90$ & $1-24$ \\
Intrusão & $90(34,6)$ & $3,9 \pm 3,26$ & $1-16$ \\
Hiperestimulação & $88(33,9)$ & $3,6 \pm 3,17$ & $1-15$ \\
IAM-UTI $\left(\mathbf{n}=\mathbf{2 9 1}^{*}\right)$ & & & \\
Tratamento & $96(33,0)$ & $5,2 \pm 2,2$ & $1-9$ \\
Ambiente & $99(34,0)$ & $5,1 \pm 1,9$ & $1-7$ \\
Emocional & $96(33,0)$ & $5,1 \pm 2,5$ & $1-11$ \\
Memórias ilusórias $\left(\mathbf{n}=61^{*}\right)$ & $4(6,6)$ & - & - \\
Reais e ilusórias & $57(93,4)$ & - & - \\
\hline
\end{tabular}

* A diferença entre o número de pacientes avaliados (138) e o observado refere-se ao número de respostas dadas pelos pacientes.

DP: desvio-padrão.

Dentre os pacientes que se recordaram de algum evento relacionado à UTI ( $n=61)$, observou-se que a maioria $(93,4 \%)$ relatou tanto memórias de fatos reais, relacionadas ao tratamento (33,0\%), ao ambiente $(34,0 \%)$ e às emocionais $(33,0 \%)$ como memórias ilusórias; e os demais $(6,6 \%)$ tiveram somente memórias ilusórias como sonhos, pesadelos e alucinações.

Quanto às recordações de fatos reais, as lembranças mais frequentes foram: a alta para a enfermaria (86,2\%), a visita da família $(79,4 \%)$, presença de confusão/agitação $(56,6 \%)$, contensão física $(54,7 \%)$ e sentir sede $(57,2 \%)$. Lembranças relacionadas ao tubo endotraqueal, à aspiração e à extubação foram relatadas por apenas $24,7 \%$ dos pacientes.

\section{DISCUSSÃO}

A avaliação dos pacientes submetidos à VM realizada três meses após a alta da UTI mostrou que poucos pacientes apresentaram sintomas de TEPT e em apenas 5,1\% o transtorno clínico foi diagnosticado. A prevalência de sintomas de TEPT encontrada entre os pacientes tratados na instituição em estudo, portanto, foi bastante inferior aos resultados encontrados na literatura, os quais apontam uma prevalência que varia entre $22 \%$ e $64 \% \%^{6,22}$.

A diferença observada na baixa incidência de pacientes com TEPT, em comparação com estudos que encontraram alta incidência, pode ser explicada pelo fato de ter sido utilizado não só o instrumento IES-R, mas também dados da entrevista psicológica, bem como devido ao fato de esse evento ter sido estudado em períodos distintos, com diferentes instrumentos, metodologias e coortes de pacientes ${ }^{3,23-26}$. A opção por estudar apenas os pacientes submetidos à VM associada a de excluir aqueles com transtorno psiquiátrico prévio pode ter sido responsável pela diferença dos resultados de outros estudos.

Estudos $^{22,23}$ que investigaram a presença de sintomas de TEPT em pacientes de UTI a partir de entrevista clínica e adotando os critérios do DSM-IV, tal como ocorreu com o presente estudo que realizou a confirmação do diagnóstico clínico com dados da entrevista psicológica, encontraram prevalência mais baixa do transtorno do que aqueles que usaram instrumentos de autorrelato.

Observou-se associação significativa entre faixa etária abaixo dos 50 anos e a presença de sintomas do TEPT. Outros estudos já haviam constatado que idade jovem é um fator de risco para o desenvolvimento de sintomas do TEPT ${ }^{3,7}$. Estudo 2 realizado com pacientes submetidos à cirurgia cardíaca e à VM identificou que após 50 anos de idade os pacientes apresentaram menos sintomas de TEPT.

No entanto, não foram observadas diferenças estatisticamente significativas entre os grupos com e sem sintomas de TEPT em relação aos fatores: sexo, causa de admissão, tempo de UTI, nível e tempo de sedação e memórias de UTI. Quanto a essas variáveis, não é possível afirmar que o grupo de pacientes com sintomas de TEPT é diferente daquele que não apresentou tais sintomas. Infere-se que a falta de significância estatística pode ter se dado em virtude do reduzido número de pacientes que apresentou sintomas do transtorno.

Apesar de neste estudo não terem sido observadas correlações significativas entre a presença de sintomas de TEPT e alguns fatores de risco já identificados em outros estudos ${ }^{2,5,8}$, quando os sete pacientes diagnosticados com TEPT foram analisados mais profundamente, constatou-se que a maioria era do sexo feminino; o tempo de UTI e VM foi superior a dois dias e esses pacientes tiveram uma longa permanência na enfermaria. Resultados da literatura têm demonstrado que pacientes jovens, do sexo feminino, com longa permanência na UTI e na enfermaria e submetidos à VM prolongada apresentaram incidência maior desse transtorno ${ }^{2,7,8}$.

Todos os pacientes diagnosticados apresentaram sintomas de depressão e/ou ansiedade, observando-se uma re- 
lação significativa entre sintomas de ansiedade e a presença de TEPT ( $p=0,0006$ ). Resultados de alguns estudos ${ }^{4,8,26}$ demonstraram que os pacientes com TEPT apresentam risco maior de desenvolver outras desordens mentais, a exemplo da ansiedade e depressão, em que, se não tratadas adequadamente, em mais de um terço dos casos os pacientes não se recuperam.

Dos 20 pacientes com sintomas de TEPT, seis apresentaram somente memórias de fatos reais, 10 relataram memórias de fatos reais e de ilusão e quatro não relataram nenhuma lembrança desse período.

O tipo de memória relatada não apresentou relação com a presença de sintomas do TEPT. Pacientes que relataram tanto memórias de fatos reais quanto ilusórias apresentaram sintomas do transtorno. Ao contrário do que tem sido descrito na literatura ${ }^{3,8}$, a presença de memórias de fatos reais parece não ter sido um fator de proteção contra o desenvolvimento de sintomas relacionados ao TEPT. Resultados de outro estudo ${ }^{15}$ mostraram que pacientes que não tinham recordações de fatos reais de sua permanência na UTI, mas apenas memórias ilusórias, apresentaram altas taxas de TEPT. Essa discrepância pode ser explicada a partir de alguns fatores: 1) no estudo citado ${ }^{15}$ as avaliações das memórias de UTI e do TEPT foram realizadas respectivamente, em duas e oito semanas após a alta da UTI, e no presente estudo elas foram avaliadas simultaneamente três meses após a alta da UTI; 2) naquele estudo ${ }^{15}$, a proporção de pacientes que apresentou memórias ilusórias foi de $20 \%$ contra 6,6\% no presente estudo, e estes não apresentaram sintomas do TEPT. E, por fim, as diferenças relacionadas às práticas e características estruturais das UTIs podem resultar nas diferenças observadas.

Embora o TEPT seja debilitante, uma vez diagnosticado, o tratamento envolve uma combinação de métodos desde a utilização de psicofármacos associados à psicoterapia até abordagens psicoeducacionais ${ }^{27}$. Em um estudo ${ }^{28}$ de intervenção cognitiva, mediante a aplicação de um manual educativo que continha sugestões de enfrentamento perante o aparecimento de sintomas do TEPT, ansiedade e depressão, observou-se uma diminuição dos sintomas referentes a esses transtornos. Outro estudo ${ }^{29}$ que investigou os efeitos psicológicos do procedimento de interrupção diária da sedação constatou significativa diminuição na sintomatologia de TEPT.

Dos agrupamentos de sintomas que compõem o TEPT, os pensamentos de evitação/entorpecimento foram os mais referidos pelos pacientes. Tais sintomas podem ser considerados como estratégias emocionais, cognitivas e comportamentais para minimizar o desgaste provocado pelo evento ${ }^{27}$. Os sintomas desse grupo caracterizam-se por esforços despendidos pelos pacientes para evitar pensamentos, sensações, atividades, pessoas e locais relacionados ao evento traumático. Os resultados de um estudo ${ }^{15}$ referente ao grupo de sintomas do TEPT apresentados pelos pacientes foram semelhantes aos encontrados no presente estudo.
Descrições de memórias de fatos reais, como a visita da família, sentir-se confuso e agitado, de ter ficado com as mãos e pés amarrados foram as mais citadas pelos participantes.

Já as memórias de ilusões, relatadas por 44,2\% dos pacientes, foram descritas como fonte de desconforto e, na maioria das vezes, estavam relacionadas com fragmentos de situações vividas durante a internação na UTI. Esses dados são consistentes com os resultados encontrados na literatura, demonstrando uma variação entre 44\% e 77\% de recordações de memórias ilusórias ${ }^{3,8,15}$.

Presença de sentimentos de impotência, medo, insegurança e perda de controle também esteve presente nos relatos dos pacientes avaliados, e tais experiências foram vivenciadas de forma muito real, gerando ansiedade, medo e até mesmo levando o paciente a exibir alterações comportamentais, a exemplo de agitação psicomotora e confusão mental.

Algumas limitações devem ser ponderadas. 1) Não foram excluídos pacientes vítimas de traumatismo cranioencefálico (TCE), sendo essa uma causa de admissão frequente na UTI estudada. No entanto, sabe-se que o TCE, dependendo da sua extensão, resulta em deficiências e incapacidades que se caracterizam por ocasionar perdas ou alterações na memória. 2) Alguns pacientes deixaram de ser avaliados por causa das condições clínicas desfavoráveis após a alta, bem como pelo não comparecimento na segunda avaliação. 3) Embora pacientes com histórico de doença mental prévia apresentem maior risco em desenvolver o TEPT, optou-se por excluir esses pacientes em virtude da falta de registro mais detalhado em prontuário. Cabe salientar, no entanto, que apenas três pacientes foram excluídos do estudo por apresentarem diagnóstico prévio de transtorno mental. E, por fim, não foi avaliada a presença de eventos traumáticos antes da internação na UTI, bem como internações anteriores. Portanto, a frequência de TEPT pode ter sido subestimada e os dados finais podem apresentar distorções.

\section{CONCLUSÃO}

O estudo demonstrou que para alguns pacientes a internação em UTI pode se constituir em um evento traumático, levando-os a desenvolver desordens emocionais. Poucos pacientes preencheram todos os critérios para o diagnóstico de TEPT, indicando que a grande maioria deles apresentou apenas sintomatologia relacionada ao TEPT. Pacientes com idade abaixo de 50 anos foram os mais acometidos com sintomas do TEPT, três meses após a alta da UTI. Assim, a identificação precoce desses sintomas e o tratamento adequado poderão trazer benefícios, tanto para a reabilitação física do paciente quanto para a prevenção de transtornos emocionais crônicos subsequentes. Portanto, o conhecimento de equipes de saúde na identificação do TEPT em pacientes de 
UTI e no desenvolvimento de estratégias de intervenção é recomendado.

\section{CONFLITOS DE INTERESSE}

Os autores declaram não existir quaisquer conflitos de interesse.

\section{REFERÊNCIAS}

1. Fontaine DK, Briggs LP, Pope-Smith B. Designing humanistic critical care environments. Crit Care Nurs. 2001;24:21-35.

2. Girard TD, Shintani AK, Jackson JC, Gordon MS, Pun BT, Henderson MS, et al. Risk factors for pos-traumatic stress disorder symptoms following critical illness requiring mechanical ventilation: a prospective cohort study. Crit Care. 2007;41(1):118.

3. Cuthbertson BH, Hull A, Strachan M, Scott J. Post-traumatic stress disorder after critical illness requiring general intensive care. Intensive Care Med. 2004;30:450-5.

4. Rattray JE, Johnston M, Wildsmith JA. Predictors of emotional outcomes of intensive care. Anaesthesia. 2005;60:1085-92.

5. Granja C, Gomes E, Amaro A, Ribeiro 0, Jones C, Carneiro A, et al. Understanding posttraumatic stress disorder-related symptoms after critical care: the early illness amnesia hypothesis. Crit Care Med. 2008;36(10):2801-9.

6. Griffiths J, Fortune G, Barber V, Young D. The prevalence of post-traumatic stress disorder in survivors of ICU treatment: a systematic review. Intensive Care Med. 2007;33(9):1506-18.

7. Scragg P, Jones A, Fauvel N. Psychological problems following ICU treatment. Anaesthesia. 2001:56:9-14.

8. Jackson JC, Hart RP, Gordon MS, Hopkins RO, Girard TD, Ely EW. Posttraumatic stress disorder and posttraumatic stress symptoms following critical illness in medical ICU patients: Assessing the magnitude of the problem. Crit Care Med. 2007;11(1):R27.

9. Jones C, Backman C, Capuzzo M, Flaatten H, Rylander C, Griffiths RD. Precipitants of post-traumatic stress disorder following intensive care: a hypothesis generating study of diversity in care. Intensive Care Med. 2007;33:978-85

10. American Psychiatry Association. Manual de diagnóstico e estatística de transtornos mentais: DSM-IV-TR (4a ed., texto revisto). Lisboa: Climepsi; 2002.

11. World Health Organization. The CID-10 Classification of Mental and Behavioural Disorders: Diagnostic Criteria for Research. Geneva: WHO; 1993.

12. Caminha RM (Org.). Transtornos do estresse pós-traumático (TEPT): da neurobiologia à terapia cognitiva. São Paulo: Casa do Psicólogo; 2005.
13. Rattray JE, Hull AM. Emotional outcome after intensive care: literature review. J Adv Nurs. 2008;64:2-13.

14. Ringdal M, Plos K, Lundberg D, Johansson L, Bergbom I. Outcome after injury: memories, health-related quality of life, anxiety, and symptoms of depression after intensive care. J Trauma. 2009;66:1226-33.

15. Jones C, Giffiths RD, Humphris G, Skirrow PM. Memory, delusions, and the development of acute posttraumatic stress disorder-related symptoms after intensive care. Crit Care Med. 2001;29:573-80

16. Caiuby AVS, Lacerda SS, Quintana MI, Torii TS, Andreoli SB. Adaptação transcultural da versão brasileira da Escala do Impacto do Evento - Revisada (IES-R). Cad Saúde Pública. 2012;28(3):597-603.

17. Costa JB, Marcon SS. Elaboração e avaliação de um instrumento para identificar memórias referentes à unidade de terapia intensiva. J Bras Psiquiatr. 2009;58(4):223-30.

18. Weiss DS, Marmar CR. The Impact of Event Scale - revised. In: Wilson JP, Keane TM (eds.). Assessing psychological trauma and PTSD. New York: Guilford; 1997. p. 399-411.

19. Jones C, Humphris G, Griffiths RD. Preliminary validation of the ICUM tool: a tool for assessing memory of the intensive care experience. Clin Intensive Care. 2000;11:251-5.

20. Creamer M, Bell R, Failla S. Psychometric properties of the Impact of Event Scale-Revised. Behav Res Ther. 2003;41:1489-96.

21. Joseph S. Psychometric evaluation of Horowitz's Impact of Event scale: a review. J Trauma Stress. 2000;13:101-13.

22. Schnyder U, Moergeli H, Klaghofer R, Buddeberg C. Incidence and prediction of posttraumatic stress disorder symptoms in severely injured accident victims. Am J Psychiatry. 2001;158:594-9.

23. Schnyder U, Morgeli H, Nigg C, Klaghofer R, Renner N, Trentz 0, et al. Early psychological reactions to life-threatening injuries. Crit Care Med. 2000;28:86-92.

24. Capuzzo M, Valpondi V, Cingolani E, Gianstefani G, De Luca S, Grassi L, et al. Posttraumatic stress disorder-related symptoms after intensive care. Minerva Anaesthesiol. 2005;71:167-79.

25. Kapfhammer HP, Rothenhausler HB, Krauseneck T, Stoll C, Schelling G. Posttraumatic stress disorder and health-related quality of life in long-term survivors of acute respiratory distress syndrome. Am J Psychiatry. 2004;161:45-52

26. Sukantarat K, Greer S, Brett S, Williamson R. Physical and psychological sequelae of critical illness. Br J Health Psychol. 2007;12:65-74.

27. Friedman M. Transtorno de estresse agudo e pós-traumático: as mais recentes estratégias de avaliação e tratamento. $4^{\mathrm{a}}$ ed. Porto Alegre: Artmed; 2009.

28. Jones C, Skirrow PM, Griffiths RD, Humphris GH, Ingleby S, Eddleston J, et al. Rehabilitation after critical illness: a randomized trial. Crit Care Med. 2003;31(10):2456-61.

29. Kress JP, Pohlman AS, O'Connor MF, Hall JB. Daily interruption of sedative infusions in critically ill patients undergoing mechanical ventilation. N Engl J Med. 2000;342:1471-7. 